\title{
THE QUALITATIVE ANALYSIS OF A DYNAMICAL SYSTEM OF DIFFERENTIAL EQUATIONS ARISING FROM THE STUDY OF MULTILAYER SCALES ON PURE METALS II
}

\author{
R. L. BAKER
}

(Communicated by David Sharp)

\begin{abstract}
We provide a qualitative analysis of the $n$-dimensional dynamical system:

$$
\dot{q}_{i}=-\sum_{j=1}^{n} \frac{a_{i j}}{q_{j}^{k}}, \quad q_{i}(t)>0, \quad i=1, \ldots, n,
$$

where $k$ is an arbitrary positive integer. Under mild algebraic conditions on the constant matrix $A=\left(a_{i j}\right)$, we show that every solution $\mathbf{q}(t), t \in[0, a)$, extends to a solution on $[0,+\infty)$, such that $\lim _{t \rightarrow+\infty} q_{i}(t)=+\infty$, for $i=$ $1, \ldots, n$. Moreover, the difference between any two solutions approaches 0 as $t \rightarrow+\infty$. We then use this result to give a complete qualitative analysis of a 3-dimensional dynamical system introduced by F. Gesmundo and F. Viani in modeling parabolic growth of three-oxide scales on pure metals.
\end{abstract}

\section{INTRODUCTION}

A metal oxide is a compound containing oxygen and metal. For instance, common rust is caused by the oxidation of metal. Certain pure metals can form different oxides, and oxidation of such metals produces a multilayer oxide scale on the metal, where the oxide layer containing the highest concentration of metal is in contact with the surface of the metal, while the oxide layer containing the highest concentration of oxygen is in contact with the gas or oxygen to which the surface of the metal is exposed. In the article [2], F. Gesmundo and F. Viani analysed the parabolic growth of three-layer oxide scales on those metals which can form three oxides. They obtained the following nonlinear three-dimensional dynamical system as a model for the growth of such scales:

$$
\begin{aligned}
& \dot{q}_{1}=\mu \frac{K_{1}}{2 q_{1}}-\frac{\mu-1}{\mu} \frac{K_{2}}{2 q_{2}}, \\
& \dot{q}_{2}=-\mu \frac{K_{1}}{2 q_{1}}+\left(\frac{\mu-1}{\mu}+\frac{\nu}{\mu}\right) \frac{K_{2}}{2 q_{2}}-\frac{\nu-1}{\nu} \frac{K_{3}}{2 q_{3}}, \\
& \dot{q}_{3}=-\frac{\nu}{\mu} \frac{K_{2}}{2 q_{2}}+\frac{K_{3}}{2 q_{3}} .
\end{aligned}
$$

Received by the editors June 20, 1994 and, in revised form, June 16, 1997.

1991 Mathematics Subject Classification. Primary 34C35, 70K05.

Key words and phrases. Differential equations, dynamical systems, nonlinear dynamical systems, cooperative dynamical systems. 
Here $K_{i}>0(i=1,2,3)$ are rate constants, $\mu, \nu$ are parameters, and $q_{i}>0$ is the weight of oxygen contained in oxide $i$ per unit area.

In the paper [1], H. C. Akuezue, M. W. Hirsch, and the author of the present paper studied the following $n$-dimensional nonlinear dynamical system:

$$
\dot{q}_{i}=-\sum_{j=1}^{n} \frac{a_{i j}}{q_{j}}, \quad q_{i}(t)>0, \quad i=1, \ldots, n .
$$

In that paper we established that under mild algebraic conditions on the real $n \times n$ matrix $A=\left(a_{i j}\right)$, the trajectories of (1.2) are well-behaved in the sense that every solution $\mathbf{q}=\left(q_{1}, \ldots, q_{n}\right):[0, a] \rightarrow \mathbf{R}^{n}, 0<a<+\infty$, can be extended to a solution on $[0,+\infty)$, such that $\lim _{t \rightarrow+\infty} q_{i}(t)=+\infty, i=1, \ldots, n$; moreover, the difference between any two solutions is bounded as a function of $t$. Finally, if $A$ is also irreducible and tridiagonal, then all solutions are eventually monotone increasing on $[0,+\infty)$. We then used this information about (1.2) to obtain a partial qualitative analysis of the dynamical system (1.1). We obtained the result that if $\mu, \nu>1$, then (1.1) has a unique parabolic solution of the form $q_{i}(t)=c_{i} \sqrt{t}, c_{i}>0, t>0$, $i=1,2,3$. We then conjectured that every solution of (1.1) in $\mathbf{R}_{++}^{3}$ is attracted to this parabolic solution, where, for $n$ any positive integer, we define

$$
\mathbf{R}_{++}^{n}=\left\{\left(x_{1}, \ldots, x_{n}\right) \in \mathbf{R}^{n} \mid x_{i}>0, i=1, \ldots, n\right\} .
$$

In the present paper, we study equation (1.2) as a special case of the more general $n$-dimensional nonlinear system:

$$
\dot{q}_{i}=-\sum_{j=1}^{n} \frac{a_{i j}}{q_{j}^{k}}, \quad q_{i}(t)>0, \quad i=1, \ldots, n, t \in[0,+\infty) .
$$

Here, $k \geq 1$ is an arbitrary positive integer and $A=\left(a_{i j}\right)$ is a real $n \times n$ matrix. We show that if the matrix $A$ satisfies the same algebraic conditions mentioned above in relation to (1.2), then every solution $\mathbf{q}=\left(q_{1}, \ldots, q_{n}\right):[0, a] \rightarrow \mathbf{R}_{++}^{n}$, $0<a<+\infty$, of (1.3) can be uniquely extended to a solution on $[0,+\infty)$, such that $\lim _{t \rightarrow+\infty} q_{i}(t)=+\infty, i=1, \ldots, n$. Furthermore, the difference between any two solutions approaches 0 as $t \rightarrow+\infty$. Finally, if $A$ is also irreducible and tridiagonal, then all solutions are eventually monotone increasing on $[0,+\infty)$. We then use these results to confirm the conjecture in [1] that all solutions of (1.1) in $\mathbf{R}_{++}^{3}$ are attracted to the parabolic solution of (1.1).

Now consider the following dynamical system, which we dub, "the generalized Lotka-Volterra equations" [4, p. 3]:

$$
\dot{p}_{i}=p_{i}^{d} \sum_{j=1}^{n} a_{i j} p_{j}^{k}, \quad p_{i}(t) \geq 0, \quad i=1, \ldots, n, t \in[0,+\infty) .
$$

Here, $d \geq 1$ is an arbitrary positive number and $k \geq 1$ is an arbitrary positive integer. Observe that the change of variable $p_{i}=1 / q_{i}$ transforms (1.3) into the specific case of (1.4) where $d=2$.

We state our main result in terms of system (1.4). For $n$ a positive integer, we use the notation

$$
\mathbf{R}_{+}^{n}=\left\{\left(x_{1}, \ldots, x_{n}\right) \in \mathbf{R}^{n} \mid x_{i} \geq 0, i=1, \ldots, n\right\} .
$$

We say that a real $n \times n$ matrix $A=\left(a_{i j}\right)$ is irreducible if for each distinct pair of indices $i, j$ with $1 \leq i \neq j \leq n$, there exists a finite sequence $i=k_{0}, \ldots, k_{m}=j$ such that $A_{k_{r-1}, k_{r}} \neq 0, r=1, \ldots, m$. 
Theorem I. Assume that the $n \times n$ matrix $A=\left(a_{i j}\right)$ in (1.4) satisfies the following four conditions:

(i) $\operatorname{det} A \neq 0$ and $a_{i j} \geq 0$, for $i \neq j$;

(ii) $A$ is irreducible;

(iii) for all $\mathbf{x}=\left(x_{1}, \ldots, x_{n}\right) \in \mathbf{R}_{+}^{n}$, if $x_{i} \sum_{j=1}^{n} a_{i j} x_{j}=0$ for $i=1, \ldots, n$, then $\mathbf{x}=0$

(iv) every real eigenvalue of $A$ is negative.

Then every solution of (1.4) of the form

$$
\mathbf{x}=\left(x_{1}, \ldots, x_{n}\right):[0, a] \rightarrow \mathbf{R}_{++}^{n}, \quad 0<a<+\infty,
$$

extends uniquely to a solution

$$
\mathbf{x}:[0,+\infty) \rightarrow \mathbf{R}_{++}^{n},
$$

such that

$$
\lim _{t \rightarrow+\infty} x_{i}(t)=0, \quad i=1, \ldots, n .
$$

Moreover, if the matrix $A$ is also tridiagonal, then $\mathbf{x}(t), t \in[0,+\infty)$, is eventually monotone decreasing on $[0,+\infty)$. Finally, in case $d=2$, any two solutions $\mathbf{x}(t)$, $\mathbf{y}(t), t \in[0,+\infty)$, of (1.4) in $\mathbf{R}_{++}^{n}$ satisfy

$$
x_{i}(t)-y_{i}(t)=o\left(x_{i} y_{i}\right), \quad i=1, \ldots, n .
$$

For $d=2$, the following is an immediate corollary of Theorem I.

Corollary I. Let the real $n \times n$ matrix $A=\left(a_{i j}\right)$ satisfy conditions (i)-(iv) of Theorem I. Then every solution of (1.3) of the form

$$
\mathbf{q}=\left(q_{1}, \ldots, q_{n}\right):[0, a] \rightarrow \mathbf{R}_{++}^{n}, \quad 0<a<+\infty,
$$

extends uniquely to a solution

$$
\mathbf{q}:[0,+\infty) \rightarrow \mathbf{R}_{++}^{n},
$$

such that

$$
\lim _{t \rightarrow+\infty} q_{i}(t)=+\infty, \quad i=1, \ldots, n .
$$

Moreover, if the matrix $A$ is also tridiagonal, then $\mathbf{q}(t), t \in[0,+\infty)$, is eventually monotone increasing on $[0,+\infty)$. Finally, if $\mathbf{r}(t), t \in[0,+\infty)$, is any other solution of (1.3) in $\mathbf{R}_{++}^{n}$, then we have

$$
\lim _{t \rightarrow+\infty}\|\mathbf{q}(t)-\mathbf{r}(t)\|=0 .
$$

Theorem II. Assume that in the dynamical system (1.1), we have $m, n>1$. Then every solution $\mathbf{p}:[0, a] \rightarrow \mathbf{R}_{++}^{3}, 0<a<+\infty$, of (1.1) extends uniquely to a solution $\mathbf{p}:[0,+\infty) \rightarrow \mathbf{R}_{++}^{3}$ such that $\lim _{t \rightarrow+\infty} p_{i}(t)=+\infty, i=1,2,3$, and this solution is eventually monotone strictly increasing on $[+\infty)$. Moreover, the system (1.1) has a unique parabolic solution $q_{i}(t)=c_{i} \sqrt{t}, c_{i}>0, i=1,2,3,0<t<+\infty$. Finally, if $\mathbf{p}:[0,+\infty) \rightarrow \mathbf{R}_{++}^{3}$ is any other solution of (1.1), then

$$
\lim _{t \rightarrow+\infty}\|\mathbf{p}(t)-\mathbf{q}(t)\|=0 .
$$




\section{Preliminaries}

In this section we present the background material and preliminary lemmas that we will use in the proofs of Theorem I and Theorem II.

Definition 2.1. Let $\mathbf{W} \subseteq \mathbf{R}^{n}$ be an open set and $F: \mathbf{W} \times \mathbf{R}_{+} \rightarrow \mathbf{R}^{n}$ a continuously differentiable vector field, and let $E$ be any subset of $\mathbf{W}$. Then the system

$$
\dot{x}_{i}=F_{i}\left(x_{1}, \ldots, x_{n}, t\right), \quad \mathbf{x}=\left(x_{1}, \ldots, x_{n}\right) \in E, \quad i=1, \ldots, n, t \in \mathbf{R}_{+},
$$

is said to be cooperative in $E$ if $\partial F_{i} / \partial x_{j}(\mathbf{a}) \geq 0$ for $i \neq j, \mathbf{a} \in E$.

The following lemma is the Müller-Kamke comparison principle (see Hirsch [3, Theorem 1.1]). For vectors $\mathbf{x}, \mathbf{y}$, we use the notation $\mathbf{x} \prec \mathbf{y}$ to mean that $x_{i}<y_{i}$ for $i=1, \ldots, n$; in a similar manner, we write $\mathbf{x} \preceq \mathbf{y}$ if $x_{i} \leq y_{i}$ for $i=1, \ldots, n$.

Lemma 2.2 (Müller-Kamke Comparison Principle). Let $F$ and $\mathbf{W}$ be as in Definition 2.1. Let $E$ be a convex subset of $\mathbf{W}$ having nonempty interior. Suppose that the system (2.1) is cooperative in $E$. Let $\mathbf{x}, \mathbf{y}:[a, b] \rightarrow E$ be solutions of (2.1) such that $\mathbf{x}(a) \prec \mathbf{y}(a)$ (resp., $\mathbf{x}(a) \preceq \mathbf{y}(a))$. Then $\mathbf{x}(t) \preceq \mathbf{y}(t)$ (resp., $\mathbf{x}(t) \preceq \mathbf{y}(t))$ for all $t \in[a, b]$.

The next lemma, due to J. Smillie [5] with improvements by H. Smith [6], demonstrates long-term monotonicity of solutions to autonomous cooperative dynamical systems that are irreducible and tridiagonal.

Lemma 2.3. Suppose that the system (2.1) is autonomous, cooperative, irreducible and tridiagonal in a convex set $E \subseteq \mathbf{W}$ having nonempty interior. Let $\mathbf{x}(t)$ be a solution in $E$ of (2.1) on a maximal interval of the form $[0, a), 0<a \leq+\infty$. Then each coordinate $x_{i}(t)$ is eventually monotone increasing or decreasing.

We borrow the next lemma from [1].

Lemma 2.4. Suppose that the real $n \times n$ matrix A satisfies conditions (i), (ii) and (iv) of Theorem I. Then $A$ has an eigenvector $\mathbf{v} \in \mathbf{R}_{++}^{n}$ with real eigenvalue $\lambda<0$.

Lemma 2.5. Let $F: \mathbf{R}_{++}^{n} \times \mathbf{R}_{+} \rightarrow \mathbf{R}^{n}$ be a continuously differentiable timedependent vector field on $\mathbf{R}^{n} \times \mathbf{R}_{+}$such that the system

$$
\dot{x}_{i}=F_{i}(\mathbf{x}, t)
$$

is cooperative in $\mathbf{R}_{++}^{n}$. Suppose that

(i) there exists $\mathbf{x}_{0} \in \mathbf{R}_{++}^{n}$, with $F\left(\mathbf{x}_{0}, 0\right)<\mathbf{0}$;

(ii) there exists a solution $\mathbf{y}:[0,+\infty) \rightarrow \mathbf{R}_{++}^{n}$ of (2.2) such that $\mathbf{y}(0) \preceq \mathbf{x}_{0}$.

Let $\mathbf{x}(t), t \in[0, a], 0<a<+\infty$, be any solution of $(2.2)$ in $\mathbf{R}^{n}$ such that $\mathbf{x}(0)=\mathbf{x}_{0}$. Then this solution extends to a unique, monotone decreasing solution

$$
\mathbf{x}:[0,+\infty) \rightarrow \mathbf{R}_{++}^{n},
$$

such that $\mathbf{y}(t) \preceq \mathbf{x}(t)$ for $t \in[0,+\infty)$.

Proof. Extend the solution $\mathbf{x}(t), t \in[0, a]$, to a unique maximal solution $\mathbf{x}:[0, b) \rightarrow$ $\mathbf{R}_{++}^{n}$. It follows from the Müller-Kamke comparison principle (Lemma 2.2) that this maximal solution is strictly decreasing on $[0, b)$. We claim that $b=+\infty$. Suppose that $b<+\infty$. Then $\lim _{t \rightarrow b-} \mathbf{x}(t) \in \partial \mathbf{R}_{++}^{n} \subseteq \mathbf{R}_{+}^{n}$. Therefore, there exists $1 \leq i \leq n$ such that $\lim _{t \rightarrow b-} x_{i}(t)=0$; then by the comparison principle, we have $0<y_{i}(t) \leq x_{i}(t), t \in[0, b)$; consequently, $0=\lim _{t \rightarrow b-} x_{i}(t) \geq \lim _{t \rightarrow b-} y_{i}(t)=$ $y_{i}(b)>0$. This contradiction proves the claim. The remainder of the lemma follows from the comparison principle. 
Lemma 2.6. Suppose that the matrix $A=\left(a_{i j}\right)$ in (1.4) satisfies conditions (i)(iii) of Theorem I. Then every solution of (1.4) of the form

$$
\mathbf{p}=\left(p_{1}, \ldots, p_{n}\right):[0, a] \rightarrow \mathbf{R}_{++}^{n}, \quad 0<a<+\infty,
$$

extends uniquely to a solution

$$
\mathbf{p}=\left(p_{1}, \ldots, p_{n}\right):[0,+\infty) \rightarrow \mathbf{R}_{++}^{n},
$$

such that $\lim _{t \rightarrow+\infty} p_{i}(t)=0, i=1, \ldots, n$. Consequently, every solution of (1.3) of the form

$$
\mathbf{q}=\left(q_{1}, \ldots, q_{n}\right):[0, a] \rightarrow \mathbf{R}_{++}^{n}, \quad 0<a<+\infty,
$$

extends uniquely to a solution

$$
\mathbf{q}=\left(q_{1}, \ldots, q_{n}\right):[0,+\infty) \rightarrow \mathbf{R}_{++}^{n},
$$

such that $\lim _{t \rightarrow+\infty} q_{i}(t)=+\infty, i=1, \ldots, n$.

Proof. Let $\mathbf{v}=\left(v_{1}, \ldots, v_{n}\right)>0$ be an eigenvector of $A$ given in Lemma 2.4 with eigenvalue $\lambda<0$. Let $\mathbf{u}=\left(v_{1}^{\frac{1}{k}}, \ldots, v_{n}^{\frac{1}{k}}\right)$. Chose $c>0$ so large that $c \mathbf{u} \succ \mathbf{p}_{0}$. Define the vector field $F: \mathbf{R}_{+}^{n} \rightarrow \mathbf{R}^{n}$ by

$$
F_{i}\left(x_{1}, \ldots, x_{n}\right)=x_{i}^{d} \sum_{j=1}^{n} a_{i j} x_{j}^{k}, \quad i=1, \ldots, n .
$$

Set $\mathbf{x}_{0}=c \mathbf{u}$; then we have $F_{i}\left(\mathbf{x}_{0}\right)=\lambda c^{d+k} v_{i}^{1+\frac{d}{k}}<0$, for $i=1, \ldots, n$. Also, $F_{i}(\mathbf{0})=0$.

By the comparison principle, $\mathbf{p}(t)$ is strictly decreasing on $[0, a]$, and $\mathbf{p}(0) \succeq \mathbf{0}$. Because $F(\mathbf{0})=\mathbf{0}$, we see that the constant function $\mathbf{0}$ is a solution of (1.4) in $\mathbf{R}_{+}^{n}$, and hence, because (1.4) is cooperative in $\mathbf{R}_{+}^{n}$, the comparison principle implies that $\mathbf{p}(t) \succeq \mathbf{0}, t \in[0, a]$. The usual compactness argument then implies that the solution $\mathbf{p}(t)$ extends over $[0,+\infty)$. Since $\mathbf{p}(t)$ is strictly decreasing on $[0, a]$ and bounded below by $\mathbf{0}$, we necessarily have that $\mathbf{p}(t)$ converges to an equilibrium point in $\mathbf{R}_{+}^{n}$. Condition (iii) of Theorem I implies that $\mathbf{0}$ is the only equilibrium in $\mathbf{R}_{+}^{n}$, hence we have $\lim _{t \rightarrow+\infty} \mathbf{p}(t)=\mathbf{0}$. This proves the first part of the lemma. The remainder of the lemma follows from making the substitution $p_{i}(t)=1 / q_{i}(t)$, $i=1, \ldots, n, t \in[0, a]$.

Lemma 2.7. Let $\mathbf{p}(t)=\left(p_{1}(t), \ldots, p_{n}(t)\right)$ and $\mathbf{q}(t)=\left(q_{1}(t), \ldots, q_{n}(t)\right), t \in[0,+\infty)$, be solutions of (1.3) in $\mathbf{R}_{++}^{n}$, with $\mathbf{p} \prec \mathbf{q}$. For $i=1, \ldots, n$, let $r_{i}, s_{i}, u_{i}, v_{i}, P_{i}, Q_{i}$ be defined by

$$
\begin{aligned}
r_{i} & =p_{i}-q_{i}, \quad s_{i}=\frac{1}{p_{i}}, \quad u_{i}=\frac{1}{q_{i}}, \quad v_{i}=p_{i}^{k+1}-q_{i}^{k+1}, \\
Q_{i} & =\sum_{j=1}^{k} p_{i}^{k-j} q_{i}^{j-1}, \quad P_{i}=Q_{i} / \sum_{j=1}^{k+1} p_{i}^{k+1-j} q_{i}^{j-1} .
\end{aligned}
$$

Let $\mathbf{r}, \mathbf{s}, \mathbf{u}, \mathbf{v}$ be defined by

$$
\mathbf{r}=\left(r_{1}, \ldots, r_{n}\right), \quad \mathbf{s}=\left(s_{1}, \ldots, s_{n}\right), \quad \mathbf{u}=\left(u_{1}, \ldots, u_{n}\right), \quad \mathbf{v}=\left(v_{1}, \ldots, v_{n}\right) .
$$


Then for $i=1, \ldots, n$, the function $(\mathbf{r}(t), \mathbf{s}(t), \mathbf{u}(t), \mathbf{v}(t)), t \in[0,+\infty)$, is a solution in $\mathbf{R}_{++}^{4 n}$ of the following dynamical system:

$$
\begin{aligned}
\dot{r}_{i} & =H_{i 1}(\mathbf{r}, \mathbf{s}, \mathbf{u}, \mathbf{v}, t):=\sum_{j=1}^{n} a_{i j} Q_{j}(t) r_{j} s_{j}^{k} u_{j}^{k}, \\
\dot{s}_{i} & =H_{i 2}(\mathbf{r}, \mathbf{s}, \mathbf{u}, \mathbf{v}, t):=s_{i}^{2} \sum_{j=1}^{n} a_{i j} s_{j}^{k}, \\
\dot{u}_{i} & =H_{i 3}(\mathbf{r}, \mathbf{s}, \mathbf{u}, \mathbf{v}, t):=u_{i}^{2} \sum_{j=1}^{n} a_{i j} u_{j}^{k}, \\
\dot{v}_{i} & =H_{i 4}(\mathbf{r}, \mathbf{s}, \mathbf{u}, \mathbf{v}, t) \\
& :=-(k+1)\left(\sum_{j \neq i}^{n} \frac{a_{i j}}{p_{j}^{k}(t)}\right) P_{i}(t) v_{i}+(k+1) q_{i}^{k}(t) \sum_{j \neq i}^{n} a_{i j} Q_{j}(t) r_{j} s_{j}^{k} u_{j}^{k} .
\end{aligned}
$$

Moreover, this system is cooperative in $\mathbf{R}_{++}^{4 n}$. Finally, for any vector $\left(\mathbf{r}_{0}, \mathbf{s}_{0}, \mathbf{u}_{0}, \mathbf{v}_{0}\right)$ in $\mathbf{R}_{++}^{4 n}$, there exists a vector $(\mathbf{r}, \mathbf{s}, \mathbf{u}, \mathbf{v}) \succ\left(\mathbf{r}_{0}, \mathbf{s}_{0}, \mathbf{u}_{0}, \mathbf{v}_{0}\right)$ such that

$$
H_{i j}(\mathbf{r}, \mathbf{s}, \mathbf{u}, \mathbf{v}, 0)<0, \quad i=1, \ldots, n, j=1,2,3,4 .
$$

Proof. First, note that $p_{i}^{k}-q_{i}^{k}=\left(p_{i}-q_{i}\right) Q_{i}, i=1, \ldots, n$. Using this identity, it is straightforward to prove (2.3.1). Equations (2.3.2) and (2.3.3) follow directly from the definitions. Using the identity $p_{i}^{k}-q_{i}^{k}=\left(p_{i}^{k+1}-q_{i}^{k+1}\right) P_{i}$, the details of $(2.3 .4)$ are as follows:

$$
\begin{aligned}
\dot{v}_{i} & =(k+1) p_{i}^{k} \dot{p}_{i}-(k+1) q_{i}^{k} \dot{q}_{i} \\
& =-(k+1) p_{i}^{k} \sum_{j=1}^{n} \frac{a_{i j}}{p_{j}^{k}}+(k+1) q_{i}^{k} \sum_{j=1}^{n} \frac{a_{i j}}{q_{j}^{k}} \\
& =-(k+1) a_{i i}-(k+1) \sum_{j \neq i}^{n} \frac{a_{i j} p_{i}^{k}}{p_{j}^{k}}+(k+1) a_{i i}+(k+1) \sum_{j \neq i}^{n} \frac{a_{i j} q_{i}^{k}}{q_{j}^{k}} \\
& =-(k+1) \sum_{j \neq i}^{n} a_{i j}\left(\frac{p_{i}^{k}}{p_{j}^{k}}-\frac{q_{i}^{k}}{q_{j}^{k}}\right) \\
& =-(k+1)\left(\sum_{j \neq i}^{n} \frac{a_{i j}}{p_{j}^{k}}\right)\left(p_{i}^{k}-q_{i}^{k}\right)+(k+1) q_{i}^{k} \sum_{j \neq i}^{n} a_{i j}\left(\frac{p_{j}^{k}-q_{j}^{k}}{p_{j}^{k} q_{j}^{k}}\right) \\
& =-(k+1)\left(\sum_{j \neq i}^{n} \frac{a_{i j}}{p_{j}^{k}}\right)\left(p_{i}^{k+1}-q_{i}^{k+1}\right) P_{i}+(k+1) q_{i}^{k} \sum_{j \neq i}^{n} a_{i j}\left[\frac{\left(p_{j}-q_{j}\right) Q_{j}}{p_{j}^{k} q_{j}^{k}}\right] \\
& =-(k+1)\left(\sum_{j \neq i}^{n} \frac{a_{i j}}{p_{j}^{k}}\right) P_{i} v_{i}+(k+1) q_{i}^{k} \sum_{j \neq i}^{n} a_{i j} Q_{j} r_{j} s_{j}^{k} u_{j}^{k} .
\end{aligned}
$$

Therefore (2.3.4) holds. Because $a_{i j} \geq 0$ for $i \neq j$, it is clear that the system (2.3) is cooperative in $\mathbf{R}_{++}^{n}$. 
To prove the last statement of the lemma, let $\mathbf{a}=\left(\alpha_{1}, \ldots, \alpha_{n}\right) \succ \mathbf{0}$ be an eigenvector of $A$ such that $A \mathbf{a}=\lambda \mathbf{a}$, where $\lambda<0$. By Lemma 2.4, such an $\mathbf{a}$ and $\lambda$ exist. Let $\left(\mathbf{r}_{0}, \mathbf{s}_{0}, \mathbf{u}_{0}, \mathbf{v}_{0}\right)$ be a given vector in $\mathbf{R}_{++}^{4 n}$. Select $b>0$ so large that $b \alpha_{i}^{\frac{1}{k}}>\max \left\{s_{0 i}, u_{0 i}\right\}$, for $i=1, \ldots, n$. Then choose $c>0$ so large that $c /\left[b^{2 k} \alpha_{i} Q_{i}(0)\right]>r_{0 i}$, for $i=1, \ldots, n$. Finally, pick $e>0$ so large that for $i=1, \ldots, n, e / P_{i}(0)>v_{0 i}$ and

$$
c q_{i}^{k}(0) \sum_{j \neq i}^{n} a_{i j} \alpha_{j}-e \sum_{j \neq i}^{n} \frac{a_{i j}}{p_{j}^{k}(0)}<0 .
$$

For $i=1, \ldots, n$, define $r_{i}, s_{i}, u_{i}, v_{i}$ by

$$
r_{i}=\frac{c}{b^{2 k} \alpha_{i} Q_{i}(0)}, \quad s_{i}=b \alpha_{i}^{\frac{1}{k}}, \quad u_{i}=b \alpha_{i}^{\frac{1}{k}}, \quad v_{i}=\frac{e}{P_{i}(0)}
$$

Then $(\mathbf{r}, \mathbf{s}, \mathbf{u}, \mathbf{v}) \succ\left(\mathbf{r}_{0}, \mathbf{s}_{0}, \mathbf{u}_{0}, \mathbf{v}_{0}\right)$. Moreover, for $i=1, \ldots, n$, we have

$$
\begin{aligned}
& H_{i 1}(\mathbf{r}, \ldots, \mathbf{v}, 0)=c \lambda \alpha_{i}<0, \\
& H_{i 2}(\mathbf{r}, \ldots, \mathbf{v}, 0)=b^{2+k} \lambda \alpha_{i}^{1+\frac{2}{k}}<0, \\
& H_{i 3}(\mathbf{r}, \ldots, \mathbf{v}, 0)=b^{2+k} \lambda c \alpha_{i}^{1+\frac{2}{k}}<0, \\
& H_{i 4}(\mathbf{r}, \ldots, \mathbf{v}, 0)=c q_{i}^{k}(0) \sum_{j \neq i}^{n} a_{i j} \alpha_{j}-e \sum_{j \neq i}^{n} \frac{a_{i j}}{p_{j}^{k}(0)}<0 .
\end{aligned}
$$

This proves the lemma.

Lemma 2.8. Let the $n \times n$ matrix $A=\left(a_{i j}\right)$ satisfy conditions (i), (ii), and (iii) of Theorem I. Let $\mathbf{p}(t)$ and $\mathbf{q}(t), t \in[0,+\infty)$, be solutions of $(1.3)$ in $\mathbf{R}_{++}^{n}$. Assume that $\mathbf{q}(0) \prec \mathbf{p}(0)$. Then for $i=1, \ldots, n$, we have

$$
\sup _{0 \leq t<+\infty}\left|p_{i}^{k+1}(t)-q_{i}^{k+1}(t)\right|<+\infty .
$$

Proof. For $t \in[0,+\infty)$ and $i=1, \ldots, n$, define

$$
r_{i}(t)=p_{i}(t)-q_{i}(t), \quad s_{i}(t)=\frac{1}{p_{i}(t)}, \quad u_{i}(t)=\frac{1}{q_{i}(t)}, \quad v_{i}(t)=p_{i}^{k+1}-q_{i}^{k+1} .
$$

For $d=2$, the functions $\mathbf{s}$ and $\mathbf{v}$ are solutions of the cooperative system (1.4). Hence, because $\mathbf{q}(0) \prec \mathbf{p}(0)$, we have $\mathbf{x}(0) \prec \mathbf{u}(0)$. Consequently, by the comparison principle, we have $\mathbf{0} \prec \mathbf{s}, \mathbf{u}$, from which it follows that $\mathbf{0} \prec \mathbf{r}, \mathbf{v}$. Next, for $i=1, \ldots, n$ and $j=1,2,3,4$, let $H_{i j}(\mathbf{r}, \mathbf{s}, \mathbf{u}, \mathbf{v}, t), t \in[0,+\infty)$, be defined as in Lemma 2.7. By this lemma, the function $(\mathbf{r}(t), \mathbf{s}(t), \mathbf{u}(t), \mathbf{v}(t)), t \in[0,+\infty)$, is a solution of the dynamical system (2.3) in $\mathbf{R}_{++}^{4 n}$; moreover, there exists a vector $\mathbf{x}_{0}=\left(\mathbf{r}_{0}, \mathbf{s}_{0}, \mathbf{u}_{0}, \mathbf{v}_{0}\right) \in \mathbf{R}_{++}^{4 n}$ such that $\left.\mathbf{x}_{0} \succ \mathbf{r}(0), \mathbf{s}(0), \mathbf{u}(0) \mathbf{v}(0)\right)$ and

$$
H_{i j}\left(\mathbf{r}_{0}, \ldots, \mathbf{v}_{0}, 0\right)<0, \quad i=1, \ldots, n, j=1,2,3,4 .
$$

By Lemma 2.7, (2.3) is cooperative in $\mathbf{R}_{++}^{4 n}$, and hence by Lemma 2.6, there exists a monotone decreasing $\mathbf{x}(t), t \in[0,+\infty)$, of $(2.3)$ in $\mathbf{R}_{++}^{4 n}$ such that $(\mathbf{r}(t), \mathbf{s}(t), \mathbf{u}(t)$, $\mathbf{v}(t)) \prec \mathbf{x}(t), t \in[0,+\infty)$. This proves $(2.4)$. 


\section{Proof of Theorem I}

In this section we prove Theorem I.

Lemma 3.1. Suppose that the matrix $A=\left(a_{i j}\right)$ satisfies the hypothesis of Theorem I. Let $\mathbf{r}(t)$ and $\mathbf{q}(t), t \in[0,+\infty)$, be solutions of (1.3) in $\mathbf{R}_{++}^{n}$. Then

$$
\lim _{t \rightarrow+\infty}\|\mathbf{r}(t)-\mathbf{q}(t)\|=0 .
$$

Proof. By Lemma 2.3, $\lim _{t \rightarrow+\infty} r_{i}(t)=q_{i}(t)=+\infty$, for $i=1, \ldots, n$. Therefore there exists a $t_{0}>0$ such that $r_{i}\left(t_{0}\right)>q_{i}(0)$, for $i=1, \ldots, n$. Define $p_{i}(t)=$ $r_{i}\left(t+t_{0}\right), i=1, \ldots, n$. Then $\mathbf{p}(t), t \in[0,+\infty)$, is a solution of (1.3) such that $\mathbf{p}(0) \succeq \mathbf{q}(0)$. Therefore, by the last statement of Lemma 2.7 and the Comparison Principle, the functions $p_{i}^{k+1}-q_{i}^{k+1}, i=1, \ldots, n$, are bounded on $[0,+\infty)$. Now, by Lemma 2.3 , for $i=1, \ldots, n$, we have $\lim _{t \rightarrow+\infty} p_{i}(t)=+\infty$, and $p_{i}-q_{i}=$ $\left(p_{i}^{k+1}-q_{i}^{k+1}\right) / \sum_{j=1}^{k+1} p_{i}^{k+1-j} q_{i}^{j-1}$, therefore, we have $\lim _{t \rightarrow+\infty}\left(p_{i}(t)-q_{i}(t)\right)=0$. Finally, for $1 \leq i \leq n, \lim _{t \rightarrow+\infty} \dot{r}_{i}(t)=0$, hence we have $\lim _{t \rightarrow+\infty}\left(p_{i}(t)-r_{i}(t)\right)=0$. This proves the lemma.

Proof of Theorem I. To prove Theorem I, let the $n \times n$ matrix $A=\left(a_{i j}\right)$ satisfy the hypothesis of Theorem I, and let $\mathbf{x}(t), t \in[0, a), 0<a<+\infty$, be a solution of (1.4) in $\mathbf{R}_{++}^{n}$. Then by Lemma 2.6, this solution extends uniquely to a solution

$$
\mathbf{x}:[0,+\infty) \rightarrow \mathbf{R}_{++}^{n},
$$

such that $\lim _{t \rightarrow+\infty} x_{i}(t)=0, i=1, \ldots, n$. Moreover, by Lemma 2.3, if $A$ is irreducible and tridiagonal, then each coordinate $x_{i}(t)$ is eventually monotone decreasing on $[0,+\infty)$.

Finally, consider the case where $d=2$. Let $\mathbf{y}(t), t \in[0,+\infty)$, be another solution of (1.4) in $\mathbf{R}_{++}^{n}$. For $i=1, \ldots, n$, let $p_{i}(t), q_{i}(t)$ be defined by

$$
p_{i}(t)=\frac{1}{x_{i}(t)}, \quad q_{i}(t)=\frac{1}{y_{i}(t)} ;
$$

then we have $\left[x_{i}(t)-y_{i}(t)\right] /\left[x_{i}(t) y_{i}(t)\right]=q_{i}(t)-p_{i}(t)$, and hence, by Lemma 3.1, we have $x_{i}-y_{i}=o\left(x_{i} y_{i}\right)$. This completes the proof of Theorem I.

\section{Proof of Theorem II}

In this final section we prove Theorem II. First, we need the following result from [1].

Lemma 4.1. Let $K_{1}, K_{2}, K_{3}>0$ and $\mu, \nu>1$ be given constants. Define the $3 \times 3$ matrix $A=\left(a_{i j}\right)$ by

$$
A=\left(\begin{array}{ccc}
-\frac{\mu K_{1}}{2} & \frac{\mu-1}{\mu} \frac{K_{2}}{2} & 0 \\
\frac{\mu K_{1}}{2} & -\left[\frac{\mu-1}{\mu}+\frac{\nu}{\mu} \frac{K_{2}}{2} \frac{\nu-1}{\nu}\right] & \frac{K_{3}}{2} \\
0 & \frac{\nu}{\mu} \frac{K_{2}}{2} & -\frac{K_{2}}{2}
\end{array}\right) .
$$

Then A satisfies conditions (i)-(iv) of Theorem I.

Proof of Theorem II. Assume that in the dynamical system (1.1), we have $\mu, \nu>1$. Let $\mathbf{p}(t)=\left(p_{1}(t), p_{2}(t), p_{3}(t)\right), t \in[0, a), 0<a<+\infty$, be a solution of (1.1) in $\mathbf{R}_{++}^{3}$. By Corollary I and Lemma 4.1, this solution extends to a unique solution $\mathbf{p}:[0,+\infty) \rightarrow \mathbf{R}_{++}^{3}$ such that $\lim _{t \rightarrow+\infty} p_{i}(t)=+\infty$ for $i=1,2,3$. 
From the results in [1], there exists a unique parabolic solution of (1.1) of the form

$$
\mathbf{q}(t)=\left(c_{1} \sqrt{t}, c_{2} \sqrt{t}, c_{3} \sqrt{t}\right), \quad c_{i}>0, \quad i=1,2,3,0<t<+\infty .
$$

From Corollary I and Lemma 4.1, we have that $\lim _{t \rightarrow+\infty}\|\mathbf{p}(t)-\mathbf{q}(t)\|=0$.

Finally, because $\mathbf{q}(t)$ is a solution of (1.1), we see that

$$
\begin{aligned}
& c_{1}=\mu \frac{K_{1}}{2 c_{1}}-\frac{\mu-1}{\mu} \frac{K_{2}}{2 c_{2}}, \\
& c_{2}=-\mu \frac{K_{1}}{2 c_{1}}+\left(\frac{\mu-1}{\mu}+\frac{\nu}{\mu}\right) \frac{K_{2}}{2 c_{2}}-\frac{\nu-1}{\nu} \frac{K_{3}}{2 c_{3}}, \\
& c_{3}=-\frac{\nu}{\mu} \frac{K_{2}}{2 c_{2}}+\frac{K_{3}}{2 c_{3}} .
\end{aligned}
$$

For $1 \leq i \leq j \leq 3$, we have $\lim _{t \rightarrow+\infty} p_{i}(t) / p_{j}(t)=c_{i} / c_{j}$, and hence by (1.1) and (4.1), we see that $\dot{p}_{i}(t)>0$ for $t$ sufficiently large, i.e., each coordinate $p_{i}(t)$ is eventually monotone strictly increasing on $[0,+\infty)$. This completes the proof of Theorem II.

\section{REFERENCES}

1. H. Akuezue, R. Baker, and M. W. Hirsch, The qualitative analysis of a dynamical system modeling the formation of multilayer scales on pure metals, SIAM J. Math. Anal. 25 (1994), 1167-1175. MR 95a:34053

2. F. Gesmundo and F. Viani, The formation of multilayer scales in the parabolic oxidation of pure metals, J. Corrosion Sci. 18 (1978), 217-230.

3. M. W. Hirsch, Systems of differential equations that are competitive or cooperative II: Convergence almost everywhere, SIAM J. Math. Anal. 16 (1985), 423-429. MR 87a:58137

4. Manfred Peschel and Werner Mende, The Predator-Prey Model, Springer-Verlag, Wien-New York, 1986. MR 87i:92056b

5. J. Smillie, Competitive and cooperative tridiagonal systems of differential equations, SIAM J. Math. Anal. 5 (1984), 530-534. MR 85g:58054

6. H. L. Smith, Periodic tridiagonal competitive and cooperative systems, SIAM J. Math. Anal. (to appear).

Department of Mathematics, University of Iowa, Iowa City, Iowa 52242

E-mail address: baker@math.uiowa.edu 